\section{EXAFS study of glass transition in $\mathrm{K}_{1-x}\left(\mathrm{NH}_{4}\right)_{x} \mathrm{I}$}

\section{Kenichiro Yagi,* Kiyoshi Sakaue and Hikaru Terauchi}

\author{
Advanced Research Center of Science and School of \\ Science, Kwansei-Gakuin University, Sanda, Hyogo \\ 669-1337 Japan
}

The iodine $K$-edge EXAFS study of $\mathrm{K}_{1-x}\left(\mathrm{NH}_{4}\right)_{x} \mathrm{I}$ mixed crystals was performed to clarify the local structure of the dipole glass associated with the random reorientational freezing of ammonium ions. The pair-distribution function of the nearest iodine-iodine ions is strongly reduced with increasing ammonium concentration. The reduction originates in the local distortion of lattice induced by the short-range antiferroelectric order of ammonium ions. We discuss the relationship between the reorientational freezing of ammonium ions and the local distortion of lattice in comparison with the results of X-ray diffraction measurements.

Keywords: EXAFS; glass transition; $\mathrm{K}_{1-x}\left(\mathrm{NH}_{4}\right)_{x} \mathrm{I}$.

\section{Introduction}

Potassium ammonium iodide, $\mathrm{K}_{1-x}\left(\mathrm{NH}_{4}\right)_{x} \mathrm{I}$, is one of the materials forming the dipole glass, associated with the random reorientational freezing of ammonium ions (Bostoen et al., 1989, Berret et al., 1992). Pure ammonium iodide is well known to undergo a drastic first-order phase transition from $\mathrm{NaCl}$-type structure to $\mathrm{CsCl}$-type one. The phase transition is suppressed by replacing ammonium ions with potassium ones, and disappears below the ammonium concentration of about $x \approx 0.7$. Further dilution of ammonium concentration leads the system to form the dipole glass, characterized by the short-range orientational order of ammonium ions (Berret et al., 1992). In the NaCl-type structure, an ammonium ion at the center of iodine octahedron is distorted from the general $T_{d}$ symmetry to $\mathrm{C}_{3 V}$ one, because of the symmetry mismatch against the octahedral environment formed by the nearest six iodine ions. The dipole moment of about $1.4 \mathrm{D}$ accompanied by the distortion lies along a three-fold axis of the ammonium ion that corresponds to the $\langle 111\rangle$ axis of fcc lattice (Fehst et al., 1990). Below the glass transition temperature $T_{\mathrm{g}}$, the reorientation of the three-fold axis of ammonium ions freezes along one of the eight equivalent $\langle 111\rangle$ directions. Our previous X-ray diffraction study revealed that the local distortion of lattice is induced by the reorientational freezing of ammonium ions (Umeki et al., 1994, Yagi et al., 1996). The strong diffuse scattering appears below $T_{\mathrm{g}}$ around the reciprocal lattice points where the Bragg reflections are forbidden by the fcc symmetry, which indicates that the local distortion breaks the fcc symmetry.

In order to obtain additional information about the local structure, we have studied the glass transition in $\mathrm{K}_{1-x}\left(\mathrm{NH}_{4}\right)_{x} \mathrm{I}$ by means of EXAFS measurements. Our previous iodine $L_{\mathrm{I}}$-edge EXAFS study of this compound revealed that the amplitude of the nearest iodine-iodine pair-distribution is strongly reduced with increasing ammonium concentration, which indicates the local distortion of lattice (Yagi et al., 1999). However, because of the small magnitude of iodine $L_{\mathrm{I}}$-edge, it was very difficult to carry out the quantitative analysis, especially at high temperatures. In this paper, we report results of the iodine $K$-edge EXAFS measurements of $\mathrm{K}_{1-x}\left(\mathrm{NH}_{4}\right)_{x} \mathrm{I}$, and discuss the relationship between the reorientational freezing of ammonium ions and the local distortion of lattice in comparison with the results of X-ray diffuse scattering measurements.

\section{Experimental}

The iodine $K$-edge EXAFS spectra $\left(E_{0}=33.169 \mathrm{keV}\right)$ of $\mathrm{K}_{1-x}\left(\mathrm{NH}_{4}\right)_{x} \mathrm{I}$ mixed crystals were measured at the beam line BL14A of Photon Factory (KEK, Japan) in the temperature range from $10 \mathrm{~K}$ to $300 \mathrm{~K}$. The synchrotron radiation $(2.5 \mathrm{GeV}, 300 \mathrm{~mA})$ was monochromatized by $\mathrm{Si}(553)$ double-crystals and detected using ionization chambers filled with $\operatorname{Ar}\left(I_{0}\right)$ and $\mathrm{Kr}(I)$ gases. The powder specimens of $x=0,0.48$ and 0.68 mixed with boron nitride were formed into a tablet. The glass transition temperature, $T_{\mathrm{g}}$, is of about $50 \mathrm{~K}$ for $x=0.48$ and $70 \mathrm{~K}$ for $x=0.68$. A closedcycle He-gas refrigerator was employed for the low-temperature measurement. EXAFS spectra were measured in a heating process ( $2 \mathrm{~K} / \mathrm{min}$.) with equilibration time of $10 \mathrm{~min}$. at given temperatures.

The programs of FEFF7 and UWXAFS3 were employed for the EXAFS analysis (Zabbinsky et al., 1995, Stern et al., 1995). The EXAFS spectra, $\chi(k)$, were extracted from absorption spectra using $A U T O B K$ included in $U W X A F S 3$. The radial structural function, $\phi(r)$, was obtained from the Fourier transform of $k$-weighted EXAFS spectra in the $k$-space from $3 \AA^{-1}$ to $12 \AA^{-1}$. The EXAFS

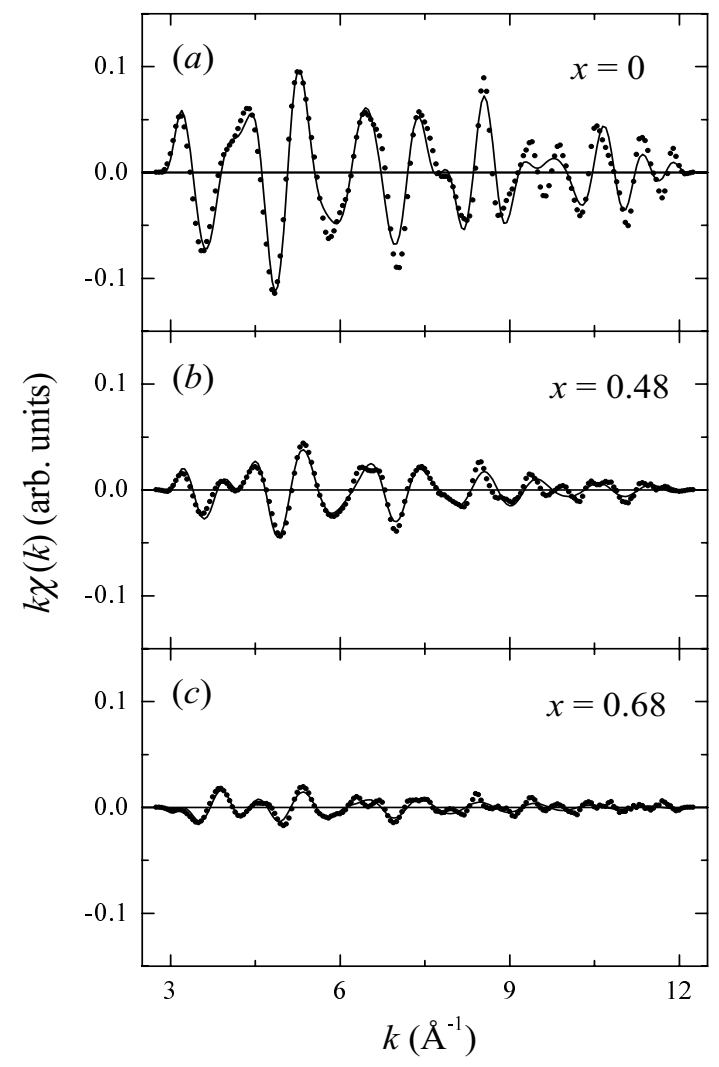

Figure 1

The $k$-weighted EXAFS spectra of $\mathrm{K}_{1-x}\left(\mathrm{NH}_{4}\right)_{x} \mathrm{I}$ mixed crystals of (a) $x=0,(b) x=0.48$ and (c) $x=0.68$ at $10 \mathrm{~K}$. The observed spectra (closed circles) were fit to the calculated ones (solid curves) using FEFF7 and UWXAFS3 programs. 
parameters were estimated using FEFFIT included in UWXAFS3 so that the observed spectra were fit to the calculated spectra in the $r$-space. The origin of photon energy was fixed at the value indicating maximum in the derivative of absorption spectra.

\section{Results and discussion}

Figure 1 shows the $k$-weighted iodine $K$-edge EXAFS spectra of $\mathrm{K}_{1-x}\left(\mathrm{NH}_{4}\right)_{x} \mathrm{I}$ mixed crystals at $10 \mathrm{~K}$. The amplitude of the EXAFS spectra is strongly reduced with increasing ammonium concentration. The observed spectra (closed circles) for each ammonium concentration are well reproduced by the calculated ones (solid curves) from the $\mathrm{NaCl}$-type structure even below $T_{\mathrm{g}}$. It is suggested from the fact that the local distortion of lattice is far from the structural change associated with the phase transition from NaCl-type structure to CsCl-type one. However, there is no doubt that the existence of ammonium ions strongly affects the local structure. Figure 2 shows absolute and imaginary parts of the radial structural function obtained from the Fourier transform of the $k$-weighted EXAFS spectra shown in Fig. 1. One should notice that the vertical axis is enlarged to make the spectra seen clearly, and that the amplitude of the spectra for $x=0$ is almost ten times as larcge as that for $x=0.68$. The observed spectra (closed circles) were reproduced by the calculated ones (solid curves) from the $\mathrm{NaCl}$-structure as well as the EXAFS spectra. The first peaks around $2.9 \AA$ correspond to the pair-distribution function (PDF) of the nearest iodinepotassium and iodine-ammonium ions, whereas the second peaks

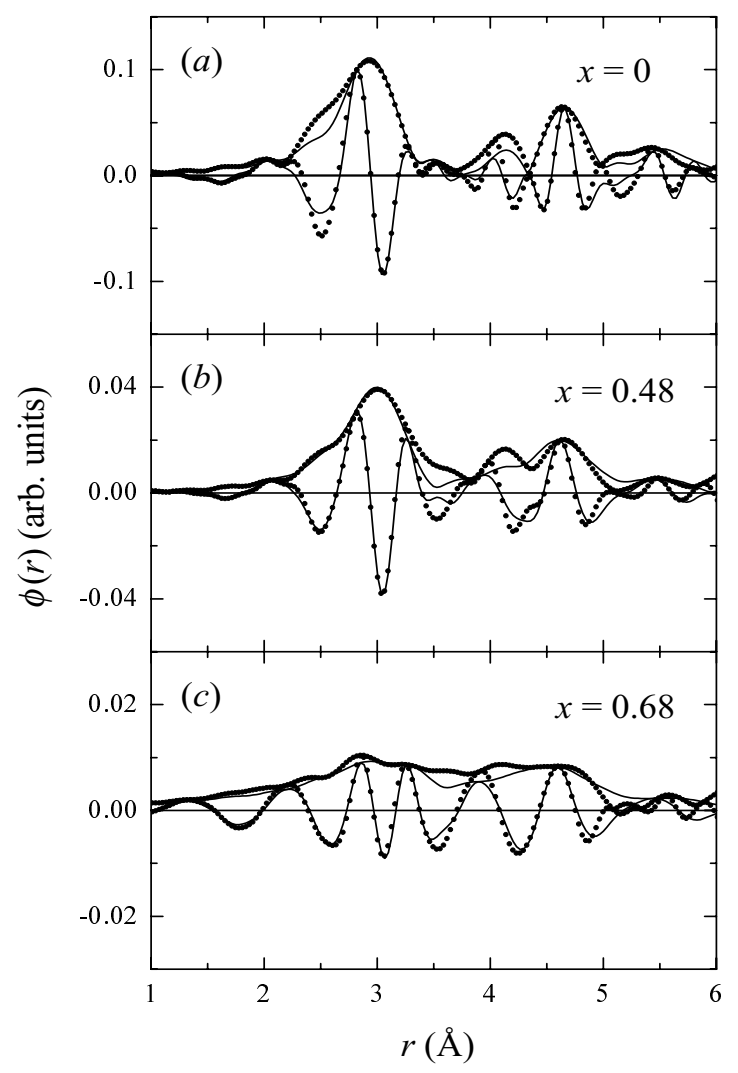

Figure 2

The Fourier transforms of the $k$-weighted iodine $K$-edge EXAFS spectra of $\mathrm{K}_{1-x}\left(\mathrm{NH}_{4}\right)_{x} \mathrm{I}$ mixed crystals of (a) $x=0,(b) x=0.48$ and (c) $x=0.68$ at $10 \mathrm{~K}$. The observed spectra (closed circles) were fit to the calculated ones (solid curves) using FEFF7 and UWXAFS3 programs. around $4.6 \AA$ correspond to that of the nearest iodine-iodine ions. The first peak is reduced with increasing ammonium concentration, because the scattering factor of ammonium ion is smaller than that of potassium ion. In addition, the second peak is also reduced with increasing ammonium concentration.

In order to discuss the results, we have to take into account the results of X-ray diffraction measurements. While the strong diffuse scattering is observed below $T_{\mathrm{g}}$, there are no anomalies in the temperature dependence of the lattice constants that obeys the Debye approximation even below $T_{\mathrm{g}}$. The Debye temperature estimated from the lattice constants is approximately fixed at $210 \mathrm{~K}$ below $x=0.7$, which indicates that the thermal expansion is almost independent on the ammonium concentration. Therefore, it is suggested that the PDF of the nearest iodine-iodine ions is reduced by the fluctuation of ions having some correlation rather than the isotropic fluctuation of ions like the thermal vibration. If the diffuse scattering is supposed to originate in such the fluctuation, the correlation should not propagate over the long-range but stay within the short-range, because the diffuse scattering exhibits the Lorentzian distribution with the correlation length less than $100 \AA$. The fluctuation seems to behave with out of phase for the nearest iodine ions and with in phase for the next-nearest ones, which can explain the results of X-ray diffraction and EXAFS measurements. The local distortion of lattice that breaks the fcc symmetry corresponds to such the fluctuation, and is expected to be induced by the coupling with the antiferroelectric short-range order of ammonium ions. We should focus upon the temperature dependence of EXAFS spectra to discuss whether the fluctuation consists of dynamical component or static one.

Figure 3 shows the temperature dependence of the DebyeWaller (DW) factor estimated from the PDF of the nearest iodineiodine ions. The DW factor, that is a measure of the mean square displacement of the ions, is increased by the reduction of the PDF. In the pure potassium iodide, $x=0$, the DW factor obeys the

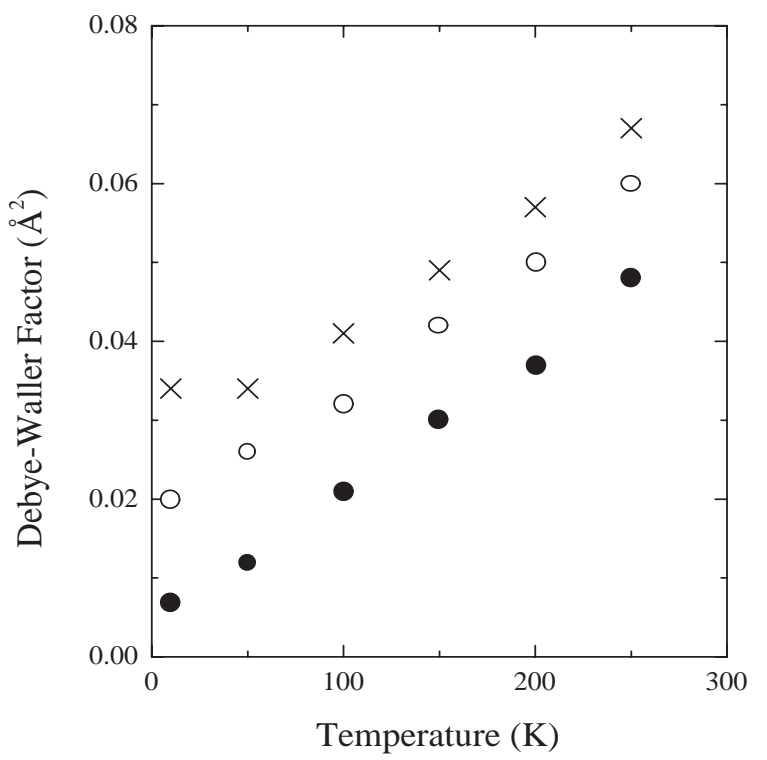

Figure 3

The temperature dependence of the Debye-Waller factor estimated from the pair-distribution of the nearest iodine ions for $x=0$ (open circles), $x=0.48$ (closed circles) and $x=0.68$ (crosses). 


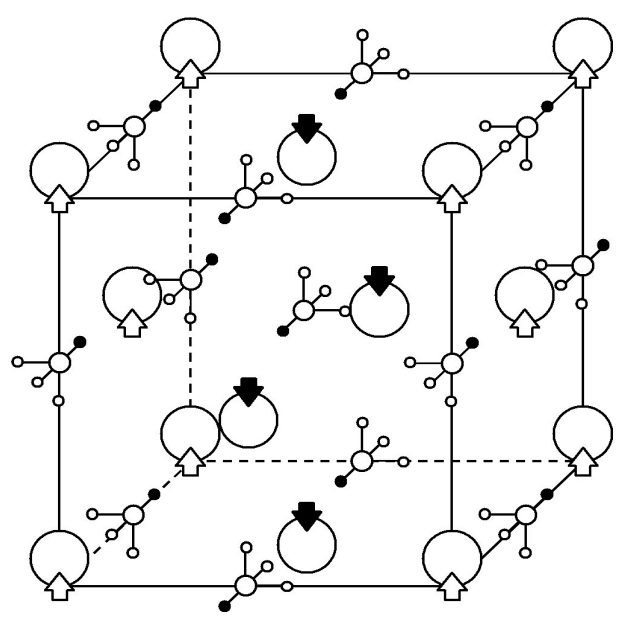

Figure 4

The schematic local structure in the glassy state determined by the analysis of X-ray and neutron diffuse scattering. The ammonium ions are randomly replaced by potassium ions.

Debye approximation within the examined temperatures, because there is no contribution to the DW factor except for the thermal vibration. The DW factor increases with increasing ammonium concentration, and the deference in the variation for each concentration remains up to high temperatures. It is strange that the temperature dependence of the Debye-Waller factor largely depends on the ammonium concentration, though the Debye temperature estimated from the lattice constants is almost independent on the concentration. The conflict should come from the fact that the DW factor reflects the PDF of the nearest iodine-iodine ions, while the lattice constants reflect that of the next-nearest iodine-iodine ions. Therefore, such the fluctuation above does not affect the lattice constants but reduces the PDF of the nearest iodine-iodine ions. If the fluctuation is induced by the coupling with the reorientation of ammonium ions, the difference in the DW factor caused by the fluctuation may remain up to high temperatures. It is speculated from the results that at high temperatures the iodine ions fluctuate so fast that the average structure shows the fcc symmetry, and that below $T_{\mathrm{g}}$ the fluctuation slows down because of the reorientational freezing of ammonium ions. When the fluctuation is adequately slow, the diffuse scattering can be observed as a evidence of the local distortion of lattice that breaks the fcc symmetry. In this study, it is difficult to determine the structural model for the local distortion, because the EXAFS spectra are strongly reduced enough to be reoughly reproduced by the NaCl-type structure. However, there is some other experimental evidence for the local distortion of lattice. We have succeeded in analyzing the X-ray and neutron diffuse scattering using the structural model shown in Fig. 4 (Yagi et al., 1998). The strong reduction of the PDF of the nearest iodine-iodine ions revealed in the EXAFS study is consistent with the relative displacement of the nearest iodine ions.

\section{Conclusion}

We performed the iodine $K$-edge EXAFS study of $\mathrm{K}_{1-x}\left(\mathrm{NH}_{4}\right)_{x} \mathrm{I}$ mixed crystals of $x=0,0.48$ and 0.68 . The EXAFS spectra are strongly reduced with increasing ammonium ion concentration. The Debye-Waller factor of the PDF of the nearest iodine-iodine ions increases gradually with temperature, as expected from the Debye approximation, and increases monotonically with ammonium concentration even above $T_{\mathrm{g}}$. It is suggested from the results that the local distortion of lattice is identified as the fluctuation of the nearest iodine ions induced by the reorientational freezing of ammonium ions, and that the fluctuation remains up to high temperatures because of the coupling with the reorientation of ammonium ions.

This work has been performed under the approval of the Photon Factory Program Advisory Committee (Proposal No. 98G127).

\section{References}

Berret, J.-F., Sauvajol, J.-L. \& Haussühl, S. (1992). J. Chem. Phys. 96, 4896-4903.

Berret, J.-F., Bostoen, C. \& Hennion, B. (1992). Phys. Rev. B46, 1374713750.

Bostoen, C., Coddens, G. \&. Wegener, W. (1989). J. Chem. Phys. 91, 63376344.

Fehst, I., Böhmer, R., Ott, W., Loidl, A., Haussühl, S. \& Bostoen, C. (1990). Phys. Rev. Lett. 64, 3139-3142.

Stern, E. A., Newville, M., Ravel, B., Yacoby, Y. \& Haskel, D. (1995). Physica B208\&209, 117-120.

Umeki, T., Yagi, K. \& Terauchi, H. (1994). J. Phys. Soc. Jpn. 63, 876-878.

Yagi, K., Umeki, U., Terauchi, H. \& Noda, Y. (1996). Physica B219\&220, 281-283.

Yagi, K., Sakaue, K., Terauchi, H. \& Noda, Y. (1998). J. Korean Phys. Soc. 32. S814-816.

Yagi, K., Sakaue, K. \& Terauchi, H. (1999). Jpn. J. Appl. Phys. 38, S152155.

Zabbinsky, S. I., Rehr, J. J., Ankudinov, A., Albers, R. C. \& Eller, M. J. (1995). Phys. Rev. B52, 2995-3009. 